\title{
A feasibility study of NIR fluorescent image- guided surgery in head and neck cancer based on the assessment of optimum surgical time as revealed through dynamic imaging
}

\author{
This article was published in the following Dove Press journal: \\ OncoTargets and Therapy \\ 5 April 2013 \\ Number of times this article has been viewed
}

\author{
Junkichi Yokoyama* \\ Mitsuhisa Fujimaki* \\ Shinichi Ohba \\ Takashi Anzai \\ Ryota Yoshii \\ Shin Ito \\ Masataka Kojima \\ Katsuhisa Ikeda \\ Department of Otolaryngology- \\ Head and Neck Surgery, Juntendo \\ University School of Medicine, Tokyo, \\ Japan \\ *These authors contributed equally \\ to this study
}

Correspondence: Junkichi Yokoyama Department of Otolaryngology-Head and Neck Surgery, Juntendo University School of Medicine, Hongo 3-I-I, Bunkyo-ku, Tokyo II3-842I, Japan $\mathrm{Tel}+8 \mid 3381331111$

Fax +8I 358407103

Email jyokoya@juntendo.ac.jp
Background: In order to minimize surgical stress and preserve organs, endoscopic or robotic surgery is often performed when conducting head and neck surgery. However, it is impossible to physically touch tumors or to observe diffusely invaded deep organs through the procedure of endoscopic or robotic surgery. In order to visualize and safely resect tumors even in these cases, we propose using an indocyanine green (ICG) fluorescence method for navigation surgery in head and neck cancer.

Objective: To determine the optimum surgical time for tumor resection after the administration of ICG based on the investigation of dynamic ICG fluorescence imaging.

Methods: Nine patients underwent dynamic ICG fluorescence imaging for 360 minutes, assessing tumor visibility at 10,30,60, 120, 180, and 360 minutes. All cases were scored according to near-infrared (NIR) fluorescence imaging visibility scored from 0 to 5 .

Results: Dynamic NIR fluorescence imaging under the HyperEye Medical System indicated that the greatest contrast in fluorescent images between tumor and normal tissue could be observed from 30 minutes to 1 hour after the administration of ICG. The optimum surgical time was determined to be between 30 minutes to 2 hours after ICG injection. These findings are particularly useful for detection and safe resection of tumors invading the parapharyngeal space.

Conclusion: ICG fluorescence imaging is effective for the detection of head and neck cancer. Preliminary findings suggest that the optimum timing for surgery is from 30 minutes to 2 hours after the ICG injection.

Keywords: indocyanine green (ICG), navigation surgery, robotic surgery, endoscopic surgery, minimally invasive surgery

\section{Introduction}

Complete resection is essential for obtaining an improved prognosis in the treatment of head and neck cancer. However, it is difficult to successfully resect advanced cases while simultaneously preserving organs. This is particularly complicated when the cancer has diffusely invaded deep organs. Tumors that cannot be physically touched or observed are often dealt with through endoscopic or robotic surgery. These procedures are highly beneficial during surgery in visualizing tumors that have invaded deep behind critical organs.

Optical imaging using near-infrared (NIR) fluorescence is a new technique that can be utilized to visually observe the operation field in real time during surgery. ${ }^{1}$ NIR fluorophores are probes that have gained immense interest in various fields 
of biomedicine. This is due to factors such as minimal interference of absorption and fluorescence found in biological samples, minimal scattering, enhanced tissue penetration depth, and low autofluorescence, which provides high-quality contrast. ${ }^{2,3}$

In this study, we used indocyanine green (ICG) with infrared camera imaging (the HyperEye Medical System [HEMS]; Mizuho Medical Co, Ltd, Tokyo, Japan) in order to investigate dynamic imaging as observed between normal and diseased tissue. To our knowledge, this is the first study to identify dynamic imaging for image-guided surgery with ICG NIR fluorescence imaging in head and neck cancer in vivo.

Our objective was to determine the optimum surgical time for tumor resection after administration of ICG based on the investigation of dynamic ICG fluorescence imaging as observed between normal and diseased tissue.

\section{Materials and methods}

Nine patients were included in this study. The numbers of oral, pharyngeal, and other cancers were two, five, and two, respectively. The number of cases of primary cancers and lymph node metastasis were five and four, respectively. Untreated cases and recurrent cases were six and three, respectively (Table 1 ).

\section{Real-time NIR fluorescent imaging}

ICG 0.5 mg/kg (Diagnogreen; Daiichi Sankyo, Tokyo, Japan) was injected via the cephalic vein. Observation of the dynamic fluorescent image was conducted with an infrared camera (HEMS). Fluorescent images were recorded at periods of 10 minutes, 30 minutes, 1 hour, 2 hours, 3 hours, and 6 hours after the initial injection. All cases were scored according to NIR fluorescence imaging visibility ${ }^{4}$ scored from 0 to 5 . A score of 0 means a lesion is not visible; a score of 1 refers to the presence of a lesion that is hardly visible; a score of 2 indicates that the contrast is weak - that is, the lesion is detectable only when its exact location is known; a score of 3 indicates that the contrast of the lesion is clearly detectable yet inferior when compared with the contrast observed in the other surrounding structures; a score of 4 indicates that the contrast of the lesion is similar to that of the other structures; and a score of 5 indicates that the contrast of the lesion dominated the image. ${ }^{5}$

Two cases were administered with ICG on two occasions. The first time was to evaluate real-time imaging, while the second was at the time of resection. This protocol was approved by the institutional review board at Juntendo University, Tokyo, Japan and all patients gave documented informed consent.

\section{Results}

\section{Real-time NIR fluorescence imaging}

After the intravenous administration of ICG under HEMS imaging, fluorescence emissions were detected throughout the primary region and the neck immediately. There was no contrast observed between the tumor and the healthy organs. Gradually, the fluorescence emissions lost brightness in normal structures. In our study, the greatest contrast between

Table I Patient characteristics and the visibility score of dynamic NIR fluorescence imaging

\begin{tabular}{|c|c|c|c|c|c|c|c|}
\hline Case & Age & Primary/lymph & Site & Rec/fresh & Size $(\mathrm{cm})$ & & \\
\hline I & 75 & Primary & Maxillary & Recurrence & 2.5 & & \\
\hline 2 & 63 & Lymph node & Larynx & Recurrence & 5.7 & & \\
\hline 3 & 45 & Primary & Oral & Fresh & 3.6 & & \\
\hline 4 & 37 & Lymph node & Oropharyx & Fresh & 2.5 & & \\
\hline 5 & 77 & Primary & Oral & Fresh & 5.2 & & \\
\hline 6 & 60 & Primary & Oropharyx & Fresh & 3.7 & & \\
\hline 7 & 52 & Lymph node & Hypopharyx & Fresh & 6.2 & & \\
\hline 8 & 55 & Primary & Oropharyx & Fresh & 6.3 & & \\
\hline 9 & 57 & Lymph node & Hypopharyx & Recurrence & 2.3 & & \\
\hline Average & 57.8 & & & & 4.22 & & \\
\hline ICG score & $0 \mathrm{~min}$ & $10 \mathrm{~min}$ & $30 \mathrm{~min}$ & $60 \mathrm{~min}$ & $120 \mathrm{~min}$ & $180 \mathrm{~min}$ & $360 \mathrm{~min}$ \\
\hline \multicolumn{8}{|l|}{ Case } \\
\hline I & 2 & 4 & 3 & 5 & 3 & 2 & 0 \\
\hline 2 & 0 & 2 & 4 & 4 & 4 & 3 & 3 \\
\hline 3 & 2 & 3 & 5 & 5 & 4 & 4 & 3 \\
\hline 4 & 0 & 1 & 4 & 4 & 4 & 3 & 3 \\
\hline 5 & I & 2 & 3 & 3 & 3 & 2 & 1 \\
\hline 6 & 2 & 3 & 5 & 3 & 3 & 3 & 2 \\
\hline 7 & 2 & 3 & 4 & 4 & 5 & 5 & 4 \\
\hline 8 & 3 & 5 & 5 & 4 & 4 & 4 & 2 \\
\hline 9 & I & 2 & 3 & 5 & 5 & 4 & 2 \\
\hline
\end{tabular}

Abbreviations: ICG, indocyanine green; lymph, lymph node; min, minutes; NIR, near infrared; rec, recurrence. 
lesions and normal structures was observed approximately 30-60 minutes after the administration of ICG (Figure 1). All nine cases were examined according to the subjective NIR fluorescence imaging visibility score. ${ }^{4}$ Scores were highest at the period of 30 minutes following ICG administration. After 6 hours, fluorescence emissions also decreased in the tumors. Twelve hours after, there was no contrast observable between the tumor and the normal structures. The results of our data are summarized in Table 1.

Two cases were resected under HEMS imaging 30-60 minutes after the administration of ICG. In one of the two cases, we observed recurrent retropharyngeal node from hypopharyngeal carcinoma (Figure 2). First, we marked the position of the tumor on pharyngeal mucosa before incision. (Figure 3A and B). We then incised the pharyngeal mucosa transorally. Even with the submucosal tumor obscured by fasciae, we were able to observe the tumor clearly under HEMS imaging (Figure 3C and D). All tumors displayed bright fluorescence emissions that clearly contrasted with the normal structures. The tumor was completely resected pathologically (Figure 4). As a result, we could remove the tumor safely and noninvasively to preserve pharyngeal functions.

\section{Discussion}

Recently, ICG fluorescence imaging has been utilized for a variety of purposes in oncologic surgery. ICG fluorescence

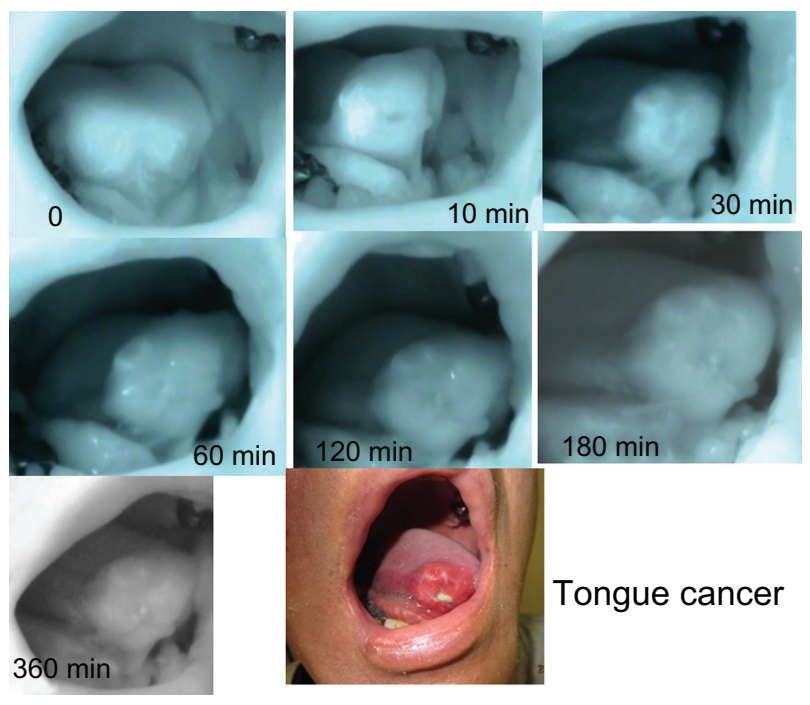

Figure I Changes in ICG fluorescence images in tongue cancer.

Notes: After ICG $(5 \mathrm{mg} / \mathrm{kg})$ administration, observation of dynamic fluorescence imaging was started with HEMS (Mizuho Medical Co, Ltd, Tokyo, Japan). At 0, 10 minutes, 30 minutes, I hour, 2 hours, 3 hours, and 6 hours after injection, bright fluorescent spots were recorded in each period. The greatest fluorescence contrast between the tumor and normal tissue was recorded after 30 minutes to I hour. Abbreviations: HEMS, HyperEye Medical System; ICG, indocyanine green.

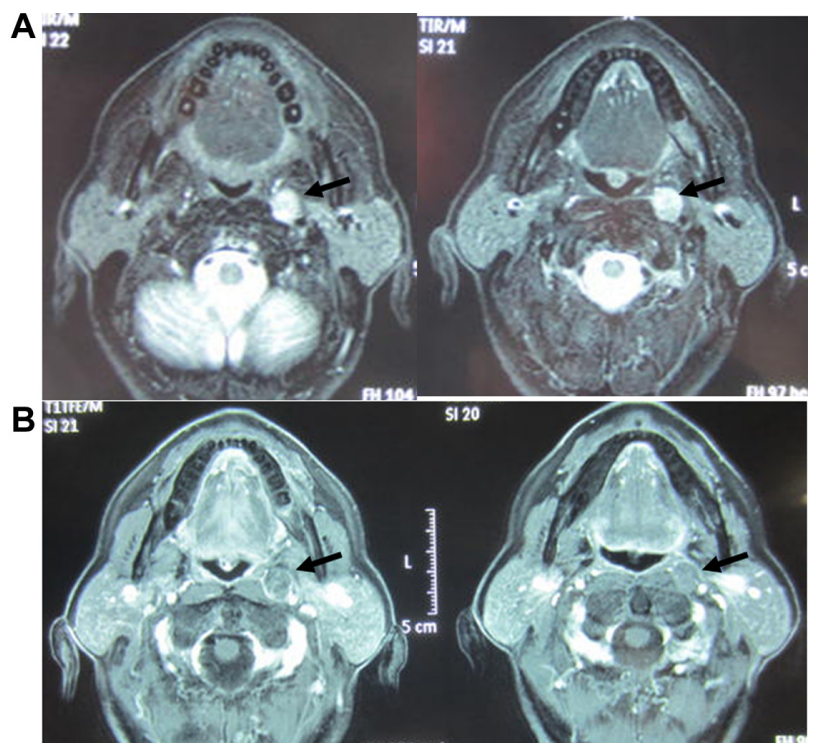

Figure 2 MRIs of a recurrent retropharyngeal node from hypopharyngeal carcinoma. (A) T2-weighted MRI shows a left recurrent retropharyngeal node (arrow). (B) MRI with fat suppression enhances only marginal zone of the tumor (arrow).

Abbreviation: MRI, magnetic resonance image.

imaging can potentially be used for (1) sentinel lymph node detection; ${ }^{6,7}(2)$ evaluation of lymphedema; ${ }^{8}$ (3) microvascular circulation of free flaps in reconstructive surgery; ${ }^{9}$ and (4) endoscopic marking of colorectal tumors. ${ }^{7}$

ICG is a water-soluble, anionic, amphiphilic tricarbocyanin probe with a hydrodynamic diameter of $1.2 \mathrm{~nm}$ and

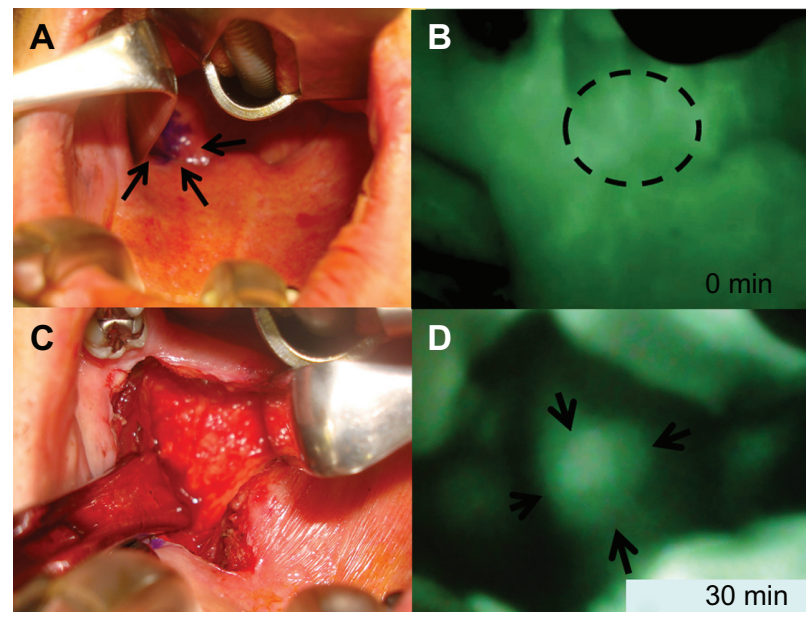

Figure 3 Examination with ICG fluorescence image for recurrent retropharyngeal node from hypopharyngeal carcinoma intraoperative findings between 0 minutes and 30 minutes after ICG injection. (A) Intrapharyngeal finding. Retropharyngeal tumor was not palpable and not visible. Tumor location marked based on ICG image (arrows). (B) ICG fluorescence image soon after ICG injection. ICG fluorescence visualizes diffusely recurrent retropharyngeal node on posterolateral oropharynx soon after ICG injection (dotted circle). (C) Pharyngeal mucosa incision. Left soft palate is incised. The tumor, covered with fasciae, cannot be observed on the white LED light 30 minutes after ICG injection. (D) ICG image at 30 minutes after ICG injection.

Note: The covered tumor can be visualized clearly under fluorescence emission. Abbreviation: ICG, indocyanine green. 


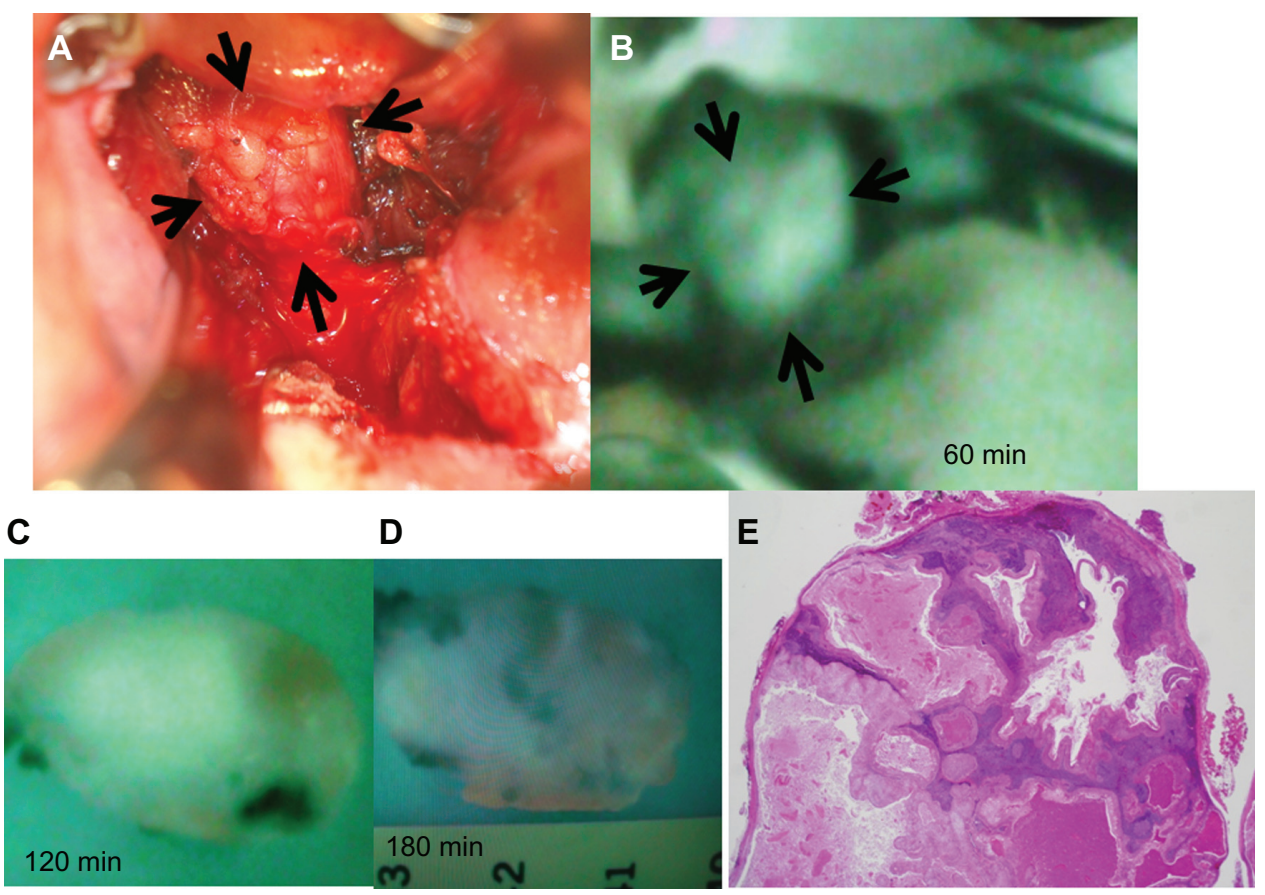

Figure 4 Examination with ICG fluorescence image for recurrent retropharyngeal node from hypopharyngeal carcinoma between 60 minutes and I80 minutes in excised specimens. (A) Intraoperative findings after 60 minutes. The tumor is observed clearly on the white LED light. (B) ICG image 60 minutes after ICG injection. ICG image visualized the tumor clearly under HEMS (Mizuho Medical Co, Ltd, Tokyo, Japan) imaging. (C) I20 minutes after ICG injection. Under HEMS imaging, the excised tumor is moderately fluorescent. (D) 180 minutes after ICG injection. Under HEMS imaging, the excised tumor is slightly fluorescent. (E) Pathological findings.

Abbreviations: HEMS, HyperEye Medical System; ICG, indocyanine green.

excitation and emission wavelengths in serum at 778 and 830 , respectively. ${ }^{10,11}$ ICG is a widely used diagnostic reagent approved for use in the examination of the hepatic function and cardiac output. ${ }^{12,13}$ After the intravenous administration of ICG, the vascular hyperpermeability of cancer towards macromolecules results in a higher fluorescence intensity in cancers observed during the extravascular phase than observed around normal tissue. In addition, ICG is cleared from the vascular compartment by the liver. ${ }^{14}$ After intravenous injection of ICG, the dye quickly binds to globulins preferentially to a1-lipoproteins within 1 to 2 seconds. ${ }^{15}$ ICG is not metabolized in the body, and hepatic elimination is the main source of clearance.

In recent years, sentinel node biopsy with ICG NIR imaging has been used extensively in head and neck cancer treatment. ${ }^{16,17}$ Several studies have reported that sentinel node biopsy with ICG NIR imaging produces results that are as good as those of a radioactive tracer; however, there have been no reports concerning image-guided surgery for the purpose of minimum invasive surgery using endoscopic and robotic surgery for the treatment of head and neck cancer. ${ }^{15,16}$ To our knowledge, this study is the first study to describe clinical application of ICG NIR dynamic imaging for examining its usefulness in head and neck cancer surgery.
Our evaluation of the dynamic imaging of ICG fluorescence emissions suggests that the contrast between ICG fluorescence tumors and normal tissue is maximally distinguished 30-60 minutes after the administration of ICG. We concluded that the optimum time for carrying out surgical procedures is between 30 minutes and 2 hours after ICG administration in head and neck cancer.

The vascular hyperpermeability of cancers toward macromolecules resulted in the saturation of fluorescence dyes in neoplastic lesions when compared to normal structures. ${ }^{12}$ During experimentation under laser scanning confocal microscopic imaging, we clearly observed the accumulation of ICG in tumor masses and no fluorescent emission in normal tissues. ${ }^{18}$ In our experimental study, we determined that the optimum surgical time was between 3 and 12 hours after the ICG injection. ${ }^{18}$ The optimum surgical time in the experimental study was significantly later than that of our study. The reason for this time lag was that not all ICG was administered via the fine tail vein, ${ }^{18}$ and some of the leaked ICG was gradually reabsorbed by veins. However, in these clinical cases, all ICG was administered via the cephalic vein over a short period of time and the optimum surgical time ranged from 30 minutes to 120 minutes. Different species' metabolism of ICG may also influence differences regarding 
the optimal surgical time using ICG fluorescence. However, it is important for us to pay attention to the fact that the accumulation of ICG may not be limited to cancerous tissues. ICG would also be expected to accumulate in inflammatory tissues and areas of surgical trauma. ${ }^{19}$

A report has indicated that ICG NIR fluorescence imaging-guided surgery is efficient in liver cancer surgery at detecting not only large cancers, but also microcancers. ${ }^{20}$ In this study, we suggest the usefulness of ICG NIR fluorescence imaging not only in liver cancer surgery, but also in head and neck cancer surgery.

In this study we used a new HEMS handheld device. The HEMS can detect tumors by ICG-enhanced images with vivid color and can be freely operated intraoperatively by surgeons. This is a high-sensitivity charge-coupled device camera and custom-made optical filter with non-Bayer color filter arrays. It can detect visible and NIR rays from 380-1200 nm without bias in color balance at 30 frames per second. ${ }^{21}$ We use the HEMS for sentinel node biopsy and fluorescent angiography in daily surgical practice. It has high sensitivity due to the color CCD camera used in ICG fluorescence imaging. ${ }^{22}$

Because of its high sensitivity, NIR imaging under HEMS can help to resect tumors that have invaded parapharyngeal space behind the internal carotid artery, internal jugular vein, and lower cranial nerves. We suggest that complete tumor resection, even for advanced cancer, can be carried out under ICG NIR fluorescent imaging without major complications, eg, causing dysfunction to important cranial nerves. Macroscopic imaging with HEMS can secure adequate tumor-free margins while minimizing surgical morbidity and preservation of function. In this study we have demonstrated a successful method of distinguishing cancer from normal tissue and optimum surgical time with HEMS in animal models. ${ }^{18}$ These results demonstrate that this method could be applied to head and neck cancer surgery. Application of endoscopic and robotic surgery for oral, pharynx, and skull base lesions enables minimally invasive surgery with superior results. We need to be able to detect tumors in deeper and invisible areas when palpation is not possible. This is required in order to resect tumors safely and can be achieved through tumor detection carried out with ICG NIR fluorescent imaging. Further investigations may lead to the development of a new, minimally invasive surgical therapy achieving both better prognosis and function preservation in head and neck cancer.

\section{Conclusion}

ICG fluorescence imaging is effective for the detection of head and neck cancer. Preliminary findings suggest that the optimum timing for surgery is from 30 minutes to 2 hours after the ICG injection. Combined with endoscopic and robotic surgery for oral, pharynx, and skull base cancers, ICG fluorescence image-guided surgery enables minimally invasive surgery with good results in head and neck cancer.

\section{Acknowledgment}

This study was supported in part by Grants-in Aid for Scientific Research from the Ministry of Education, Culture, Sports, and Technology (22591920) of Japan.

\section{Author contributions}

TA and SO conceived of the study and participated in its design and coordination. MK and RY drafted the manuscript. JY and KI were involved in revising the manuscript. All authors read and approved the final manuscript.

\section{Disclosure}

The authors report no conflicts of interest in this work.

\section{References}

1. Ogawa M, Kosaka N, Choyke PL, Kobayashi H. In vivo molecular imaging of cancer with a quenching near-infrared fluorescent probe using conjugates of monoclonal antibodies and indocyanine green. Cancer Res. 2009;69(4):1268-1272.

2. Escobedo JO, Rusin O, Lim S, Strongin RM. NIR dyes for bioimaging applications. Curr Opin Chem Biol. 2010;14(1):64-70.

3. Kirchherr AK, Briel A, Mäder K. Stabilization of indocyanine green by encapsulation within micellar systems. Mol Pharm. 2009;6(2): 480-491.

4. Poellinger A, Burock S, Grosenick D, et al. Breast cancer: early - and late-fluorescence near-infrared imaging with indocyanine green - a preliminary study. Radiology. 2011;258(2):409-416.

5. Grosenick D, Moesta KT, Möller M, et al. Time-domain scanning optical mammography: I. Recording and assessment of mammograms of 154 patients. Phys Med Biol. 2005;50(11):2429-2449.

6. Murawa D, Hirche C, Dresel S, Hünerbein M. Sentinel lymph node biopsy in breast cancer guided by indocyanine green fluorescence. $\mathrm{Br}$ J Surg. 2009;96(11):1289-1294

7. Noura S, Ohue M, Seki Y, et al. Evaluation of the lateral sentinel lymph node by indocyanine green for rectal cancer based on micrometastasis determined by reverse transcriptase polymerase chain reaction. Oncol Rep. 2008;20(4):745-750.

8. Maus EA, Tan IC, Rasmussen JC, et al. Near-infrared fluorescence imaging of lymphatics in head and neck lymphedema. Head Neck. 2012;34(3):448-453.

9. Betz CS, Zhorzel S, Schachenmayr H, et al. Endoscopic measurements of free-flap perfusion in the head and neck region using red-excited indocyanine green: preliminary results. J Plast Reconstr Aesthet Surg. 2009;62(12):1602-1608.

10. Ohnishi S, Lomnes SJ, Laurence RG, Gogbashian A, Mariani G, Frangioni JV. Organic alternatives to quantum dots for intraoperative near-infrared fluorescent sentinel lymph node mapping. Mol Imaging. 2005;4(3):172-181.

11. Gurfinkel M, Thompson AB, Ralston W, et al. Pharmacokinetics of ICG and HPPH-car for the detection of normal and tumor tissue using fluorescence, near-infrared reflectance imaging: a case study. Photochem Photobiol. 2000;72(1):94-102. 
12. Tanaka E, Chen FY, Flaumenhaft R, Graham GJ, Laurence RG, Frangioni JV. Real-time assessment of cardiac perfusion, coronary angiography, and acute intravascular thrombi using dual-channel near-infrared fluorescence imaging. J Thorac Cardiovasc Surg. 2009;138(1):133-140.

13. Deja M, Ahlers O, Macguill M, et al. Changes in hepatic blood flow during whole body hyperthermia. Int J Hyperthermia. 2010;26(2): 95-100.

14. HagenA, GrosenickD, MacdonaldR, etal.Late-fluorescencemammography assesses tumor capillary permeability and differentiates malignant from benign lesions. Opt Express. 2009;17(19):17016-17033.

15. Brouwer OR, Klop WM, Buckle T, et al. Feasibility of sentinel node biopsy in head and neck melanoma using a hybrid radioactive and fluorescent tracer. Ann Surg Oncol. 2012;19(6):1988-1994.

16. Bredell MG. Sentinel lymph node mapping by indocyanin green fluorescence imaging in oropharyngeal cancer - preliminary experience. Head Neck Oncol. 2010;2:31.

17. Baker KJ. Binding of sulfobromophthalein (BSP) sodium and indocyanine green (ICG) by plasma alpha-1 lipoproteins. Proc Soc Exp Biol Med. 1966;122(4):957-963.
18. Fujimaki M, Yokoyama J, Ohba S, et al. Dynamic imaging in determining the optimum surgical time for NIR fluorescent image-guided surgery - a preliminary study. Head Neck Oncol. 2012;4(2):50.

19. Martirosyan NL, Cavalcanti DD, Eschbacher JM, et al. Use of in vivo near-infrared laser confocal endomicroscopy with indocyanine green to detect the boundary of infiltrative tumor. J Neurosurg. 2011;115(6): 1131-1138.

20. Ishizawa T, Fukushima N, Shibahara J, et al. Real-time identification of liver cancers by using indocyanine green fluorescent imaging. Cancer. 2009;115(11):2491-2504.

21. Handa T, Katare RG, Nishimori H, et al. New device for intraoperative graft assessment: HyperEye charge-coupled device camera system. Gen Thorac Cardiovasc Surg. 2010;58(2):68-77.

22. Yokoyama J, Ito S, Ohba S, Fujimaki M, Ikeda K. A novel approach to translymphatic chemotherapy targeting sentinel lymph nodes of patients with oral cancer using intra-arterial chemotherapy - preliminary study. Head Neck Oncol. 2011;3:42.
OncoTargets and Therapy

\section{Publish your work in this journal}

OncoTargets and Therapy is an international, peer-reviewed, open access journal focusing on the pathological basis of all cancers, potential targets for therapy and treatment protocols employed to improve the management of cancer patients. The journal also focuses on the impact of management programs and new therapeutic agents and protocols on

\section{Dovepress}

patient perspectives such as quality of life, adherence and satisfaction. The manuscript management system is completely online and includes a very quick and fair peer-review system, which is all easy to use. Visit http://www.dovepress.com/testimonials.php to read real quotes from published authors.

Submit your manuscript here: http://www.dovepress.com/oncotargets-and-therapy-journal 